\title{
The Effects of Bridge Exercise on an Unstable Base of Support on Lumbar Stability and the Thickness of the Transversus Abdominis
}

\author{
Misuk Cho, PhD, $\mathrm{PT}^{1)}$, Hyewon Jeon, MS, $\mathrm{PT}^{1)^{*}}$ \\ 1) Department of Physical Therapy, Korea Nazarene University: 48 Wolbong Ro, Seobuk-gu, \\ Cheonan-Si, Chungcheongnam-do 330-718, Republic of Korea. TEL: +82 64-725-9154
}

\begin{abstract}
Purpose] We examined the effects of an abdominal drawing-in bridge exercise using a pressure biofeedback unit on different bases on the thickness of trunk and abdominal muscles, and lumbar stability. [Subjects] Thirty healthy young adults ( 2 males, 28 females) took part in this study. The subjects were randomly and equally assigned to a stable bridge exercise group and an unstable bridge exercise group. [Methods] The subjects performed bridge exercises using an abdominal drawing-in method on a stable base and on an unstable base, and changes in their abdominal muscle thickness and on the stable and on unstable bases lumbar stability were evaluated. [Results] After the intervention, the stable bridge exercise group showed a statistically significantly increased muscle thickness in the transversus abdominis, and the unstable bridge exercise group showed significantly increased muscle thicknesses of the transversus abdominis and internal obliques in static and dynamic lumbar stability. The unstable bridge exercise group showed significant increase after performing the exercise. [Conclusion] Lumbar stability exercise, with the compensation of the lumbar spine minimized, using an abdominal drawing-in method on an unstable support of base is effective and efforts to prevent the compensation may induce a greater exercise effect. Key words: Bridge exercise, Abdominal drawing-in method, Transversus abdominis
\end{abstract}

(This article was submitted Jan. 9, 2013, and was accepted Feb. 15, 2013)

\section{INTRODUCTION}

Patients with lumbar pain have greater atrophy of the deep trunk muscles than healthy subjects and they also have weakened proprioceptive senses, which trigger spinal instability, which in turn becomes the cause of recurrent lumbar pain ${ }^{1)}$. Lumbar stability is a prerequisite element that enables movement of the limbs by maintaining the spine upright in postural changes and under loading ${ }^{2}$. Muscles that contribute to lumbar stability include the transversus abdominis, and the internal, and external obliques of the abdomen, quadratus lumbrum and multifidus of the trunk posterior region, and the pelvic floor muscle ${ }^{3,4)}$. In particular, the multifidus and the transversus abdominis are activated faster than the other muscles when the human body moves, adjusting trunk balance, and the bilateral internal obliques are important for maintaining the lateral stability of the spine and spinal bending ability.

Various lumbar stability exercises have been used to maintain and improve lumbar stability through isometric contraction of the abdomen and the lumbar region. Bridge exercise, one of lumbar spine exercises, is a closed kinetic chain, weight bearing exercise, which induces contraction of the multifidus and transversus abdominis. It also improves the coordination of the large muscles of the body surface surrounding the trunk, and is effective at recovering adjust-

*To whom correspondence should be addressed.

E-mail: junhw0720@naver.com ment capabilities of the trunk $\mathrm{k}^{3,5)}$. However, excessive lumbar lordosis may occur due to compensation during bridge exercise $^{6}$. Abdominal drawing-in contracts the transversus abdominis and the internal obliques and induces concurrent contraction of the muscles during lumbar stability exercise, reducing excessive lumbar lordosis or pelvic anterior tilt that may occur during bridge exercise ${ }^{2)}$.

Recent research concerning lumbar stability exercise has used equipment such as an unstable base, a balance plate, a ball for treatment, and a sponge pad. O'Sullivan et al. ${ }^{7)}$ noted that performing stabilization exercises in a dynamic environment, for example, using a ball for treatment rather than a static environment, maximized balance ability. Page $^{8)}$ also observed that exercise using an unstable device was effective for postural control and recovery of the somatic senses.

Previous studies compared trunk stability using an abdominal drawing-in method during bridge exercise or compared trunk stability and muscle activity using bridge exercise on an unstable base of support. However, research that compares lumbar stability and the thickness of abdominal muscles by conducting bridging exercise with an abdominal drawing-in method on stable and unstable bases of support has been lacking. Accordingly, this study of support examined the effects of the abdominal drawing-in bridge exercise using a pressure biofeedback device on different bases on the thickness of trunk and abdominal muscles and lumbar stability. 


\section{SUBJECTS AND METHODS}

Thirty young adults ( 2 males, 28 females) were recruited for this study. Subjects were randomly and equally assigned to a stable bridge exercise group (SBEG) (1 male, 14 female) and an unstable bridge exercise group (UBEG) (1 male, 14 females). Those who had problems with muscles, the skeletal system, or the nervous system or had pain in the lumbus or the pelvis during ordinary activities, or could not performt bridge exercise because they had pain in the knees or ankles were excluded. The intent of this study and the content of the entire experiment were sufficiently explained to the subjects and voluntary consent was to participation was received from them. The mean $\pm \mathrm{SD}$ age, height, and weight of the SBEG were respectively $19.87 \pm 0.35$ years, $162.84 \pm 6.62 \mathrm{~cm}$, and $58.34 \pm 9.36 \mathrm{~kg}$, and the mean $\pm \mathrm{SD}$ age, height, and weight of the UBEG were respectively $20.62 \pm 0.42$ years, $\quad 162.74 \pm 4.85 \mathrm{~cm}$, and $57.37 \pm 9.73 \mathrm{~kg}$. Analysis of gender was made with the $\chi^{2}$ test, and that of age, height, and weight was conducted with the independent t-test. There were no statistically significant differences between the two groups were considered homogenous.

The subjects performed a bridge exercise using an abdominal drawing-in method on a stable base and an unstable base. In order to provide an unstable base for the bridge exercise using an abdominal drawing-in method of an Airex Balance Pad (Alcan-Airex AG, Sins, Switzerland) with a width of $50 \mathrm{~cm}$, length of $41 \mathrm{~cm}$, and height $6 \mathrm{~cm}$ was used. For the starting position of the bridge exercise, the subjects opened both arms to form an angle of about 30 degrees, with the knee joints bent at 90 degrees, and placed the palms on the base. Then with the knees and feet shoulder width, they placed the soles of the feet on the ground in parallel. The head and the neck were maintained straight with the eyes looking at the ceiling. The subjects held up the pelvis according to the tester struction of "hold up the buttocks" and maintained the posture for 15 seconds (contraction) according to the tester instruction of "maintain the position" and lowered the pelvis to the ground for 5 seconds (relaxation) according to the tester instruction of "place down". At this moment the tester directed the subjects to draw in the navel in the upper posterior direction (lumbar direction) so that the abdominal area slightly subsided. Ten repetitions of this process were considered one set and a total of six sets were conducted $^{3)}$.

This study employed ultrasonic diagnostic equipment (MysonoU5, Samsung Medison, Korea) in order to examine changes in the thicknesses of the trunk and abdominal muscles prior to and after the bridge exercise using an abdominal drawing-in method. A $12 \mathrm{MHz}$ linear probe was used and the measured area was marked during the first measurement in order to measure the same position. One tester experienced in measuring ultrasonic images conducted the measurements.

The subjects relaxed the abdominal area and comfortably lay in a supine position on the testing board. The probe was transversely placed on the top of the iliac chest, and moved toward the center of abdomen. The probe was stopped where all of the external obliques, internal obliques, and transversus abdominis were visible. The thickness of the internal obliques and transversus abdominis was measured at a point $13 \mathrm{~mm}$ moved distal from the region where the fascias of the muscles met ${ }^{9}$. For the measurement of the multifidus, the subject lay comfortably in the prone position, and the probe was placed perpendicularly on the left transverse process of the fourth lumbar spine. The thickness from the left transverse process to the surface muscles was measured ${ }^{10)}$. The subjects were instructed to abstain from talking, laughing, or chewing during the data collection period and all images were captured and measured at maximal inspiration.

In order to evaluate static and dynamic lumbar stability prior to and after the bridge exercise using an abdominal drawing-in method, contractibility of the transversus abdominis was measured using a pressure biofeedback unit (PBU) (Stabilizer, Chattanooga Group Inc., Hixson, USA) ${ }^{11)}$. The PBU is an inelastic $16.7 \times 24.0 \mathrm{~cm}$ sack that can be inflated is connected to a pressure gauge, and excessive changes in PBU pressure show that movement of the lumbar pelvic area is not being adjusted. For the measurement of static lumbar stability (SLS), subjects lay in the prone position on hard ground and the PBU was placed below the navel at the midpoint between the bilateral anterior superior iliac spines. At a PBU baseline pressure of $70 \mathrm{mmHg}$, the subject pulled in their abdomen as maximally as possible for 10 seconds without moving the lumbus or hip joint, relieving pressure in the PBU. Then, the change in the PBU was measured and recorded. For the measurement of dynamic lumbar stability (DLS), the subjects lay in the supine position. At a PBU was placed $2 \mathrm{~cm}$ below the posterior superior iliac spine vertically from the lumbar spine. With the baseline pressure of $40 \mathrm{mmHg}$, the hip and knee joints of one leg were bent and the foot was placed on the ground and the hip joint was abducted to about 45 degrees. The changes in PBU were measured and recorded when the subject returned to the starting position ${ }^{12}$. In order to minimize the sway of the trunk during measurement, folded towels were placed on both sides of the PBU.

SPSS version 12.0 (SPSS, Chicago, IL, USA) was used for the statistical analysis of the data. The independent t-test was conducted in order to compare the general characteristics of the two groups, and the paired t-test was performed in order to compare the muscle thickness and lumbar stability of each group between prior to and after the exercise of each group. The independent t-test was used in order to compare muscle thickness and lumbar stability between the groups prior to and after the exercise. The significance level was chosen as 0.05 .

\section{RESULTS}

According to the comparison of the pre-intervention and post-intervention results of the SBEG and the UBEG, only Tra was significantly different in the SBEG; however, Tra, IO, SLS, and DLS were significantly different in the UBEG $(\mathrm{p}<0.05)$ (Table 1).

According to the comparison of the results the SBEG and the UBEG at pre-intervention, and post-intervention, 
Table 1. Comparison of Tra, IO, Multifidus, SLS and DLS between pre- and postintervention in each group (mean \pm SD) (unit: Tra, IO, Multifidus-mm, SLS, DLS-mmHg)

\begin{tabular}{llcc}
\hline Category & Group & Pre-intervention & Post-intervention \\
\hline \multirow{2}{*}{ Tra } & SBEG* $^{*}$ & $0.34 \pm 0.13$ & $0.43 \pm 0.18$ \\
& UBEG* $^{*}$ & $0.30 \pm 0.19$ & $0.46 \pm 0.25$ \\
\cline { 2 - 4 } IO & SBEG & $0.51 \pm 0.20$ & $0.65 \pm 0.14$ \\
& UBEG* & $0.50 \pm 0.12$ & $0.73 \pm 0.14$ \\
\cline { 2 - 4 } Multifidus & SBEG & $2.44 \pm 0.64$ & $2.50 \pm 0.25$ \\
& UBEG & $2.54 \pm 0.44$ & $2.48 \pm 0.29$ \\
\cline { 2 - 4 } SLS & SBEG & $2.41 \pm 2.06$ & $3.25 \pm 1.42$ \\
& UBEG* & $3.41 \pm 3.14$ & $4.58 \pm 2.81$ \\
\multirow{2}{*}{ DLS } & SBEG & $2.25 \pm 0.45$ & $1.75 \pm 1.05$ \\
& UBEG* $^{*}$ & $2.33 \pm 0.98$ & $1.50 \pm 0.67$ \\
\hline
\end{tabular}

$* p<0.05$; SBEG stable bridge exercise group; UBEG unstable bridge exercise group; Tra transversus abdominis; IO internal obliquus abdominis; SLS static lumbar stability; DLS dynamic lumbar stability

Table 2. Comparison of Tra, IO, Multifidus, SLS and DLS between SBEG and UBEG $(\mathrm{mean} \pm \mathrm{SD})$

\begin{tabular}{|c|c|c|c|}
\hline & Category & SBEG & UBEG \\
\hline \multirow{5}{*}{ Pre-intervention } & Tra & $0.34 \pm 0.13$ & $0.30 \pm 0.19$ \\
\hline & $\mathrm{IO}$ & $0.51 \pm 0.20$ & $0.50 \pm 0.12$ \\
\hline & Multifidus & $2.44 \pm 0.64$ & $2.54 \pm 0.44$ \\
\hline & SLS & $2.41 \pm 2.06$ & $3.41 \pm 3.14$ \\
\hline & DLS & $2.25 \pm 0.45$ & $2.33 \pm 0.98$ \\
\hline \multirow{5}{*}{ Post-intervention } & Tra & $0.43 \pm 0.18$ & $0.46 \pm 0.25$ \\
\hline & IO & $0.65 \pm 0.14$ & $0.73 \pm 0.14$ \\
\hline & Multifidus & $2.50 \pm 0.25$ & $2.48 \pm 0.29$ \\
\hline & SLS & $3.25 \pm 1.42$ & $4.58 \pm 2.81$ \\
\hline & DLS & $1.75 \pm 1.05$ & $1.50 \pm 0.67$ \\
\hline \multirow{5}{*}{$\begin{array}{l}\text { Change between pre- } \\
\text { and post-intervention }\end{array}$} & Tra & $0.09 \pm 0.06$ & $0.15 \pm 0.19$ \\
\hline & $\mathrm{IO}$ & $0.14 \pm 0.24$ & $0.22 \pm 0.19$ \\
\hline & Multifidus & $0.06 \pm 0.81$ & $-0.06 \pm 0.63$ \\
\hline & SLS & $0.83 \pm 1.58$ & $1.16 \pm 0.93$ \\
\hline & DLS & $0.50 \pm 1.31$ & $0.83 \pm 1.19$ \\
\hline
\end{tabular}

$* \mathrm{p}<0.05$

and the change between pre- and post-intervention, none of the items were significantly different $(\mathrm{p}<0.05)$ (Table 2$)$.

\section{DISCUSSION}

This study compared the effects of bridge exercise using an abdominal drawing-in method on stable and unstable bases of support on the thicknesses of lumbar and abdominal muscles and lumbar stability.

The purpose of spinal stability exercise is to protect spinal joints from repetitive micro-damage caused by the muscles around the spine, pain from spinal instability, and degenerative spinal changes ${ }^{5}$. The abdominal drawing-in method is effective at activating abdominal muscles, in particular, the transversus abdominis ${ }^{4}$. Susan et al. ${ }^{12)}$ reported that ultrasonography of the transversus abdominis showed activation of the muscle after abdominal drawing-in exercise. Stevens et al. ${ }^{5)}$ reported that bridging exercise with the lumbar spine in the neutral position increased activity of the abdominal muscles, but did not significantly increase activity of the multifidus. This result is consistent with those of the present study which the thickness of the transversus abdominis significantly increased $(p<0.05)$ when the bridge exercise using an abdominal drawing-in method was performed on different bases. On the other hand, the changes in the thickness of the multifidus were not statistically significant. We consider this is because compensation bridge exercise using the abdominal drawing-in method increased activity of the abdominal muscles and minimizes substitutions, such as through increased lumbar lordosis, relatively reducing action by erector spinae muscles such as the multifidus. 
Cholewicki and McGill ${ }^{13)}$ observed that stability indexes of the lumbar spine during performance of different tasks were higher in tasks that placed a lot of load on the lumbar spine. When instability in the base of support increases, more muscles for stability and balance maintenance, and more load on the lumbar spine increases muscle contraction $^{14)}$. This result is consistent with those of the present study in which bridge exercise on the unstable base significantly increased $(\mathrm{p}<0.05)$ dynamic and static lumbar stability relative to bridge exercise on the unstable base. Adjusting the degree of difficulty of the bridge exercise on the unstable base, inducing greater trunk muscle activity, and improving lumbar stability may have resulted in a better exercise effect than exercise on the stable base of support.

Lumbar pain triggers fast contraction of the abdominal muscles and impairs muscle function, and compared to healthy subjects, lumbar pain patients' muscle recruitment patterns are modified ${ }^{15)}$ resulting in instability between spinal segments. Therefore, in clinical treatment of lumbar pain patients, conducting lumbar stability exercise using an abdominal drawing-in method minimizing compensation by the lumbar spine, should be effective at increasing muscle strength and the spinal instability of the lumbar region. Also efforts to prevent compensation should result in greater exercise effect.

\section{REFERENCES}

1) O'Sullivan PB, Burnett A, Floyd AN, et al.: Lumbar repositioning deficit in a specific low back pain population. Spine, 2003, 28: 1074-1079.

2) Willson JD, Dougherty CP, Ireland ML, et al.: Core stability and its relationship to lower extremity function and injury. J Am Acad Orthop Surg,
2005, 13: 316-325. [Medline]

3) Kisner C, Colby LA: Therapeutic Exercise: Foundation and techniques, 4th ed. Philadelphia F.A. Davis, 2002: pp 657-667.

4) O'Sullivan PB, Grahamslaw KM, Kendell M, et al.: The effect of different standing and sitting postures on trunk muscle activity in a pain-free population. Spine, 2002, 27: 1238-44.

5) Stevens VK, Coorevits PL, Bouche KG, et al.: The influence of specific training on trunk muscle recruitment patterns in healthy subjects during stabilization exercises. Man Ther, 2007, 12: 271-279. [Medline] [CrossRef]

6) Richardson CA, Jull GA: Muscle control-pain control. What exercises would you prescribe? Man Ther, 1995, 1: 2-10. [Medline] [CrossRef]

7) O'Sullivan PB, Phyty GD, Twomey LT, et al.: Evaluation of specific stabilizing exercise in the treatment of chronic low back pain with radiologic diagnosis of spondylolysis or spondylolisthesis. Spine, 1997, 22: 2959-2967.

8) Page P: Sensorimotor training: A "global" approach for balancetraining. K Body Mov Ther, 2006, 10: 77-84. [CrossRef]

9) Endleman I, Critchley DJ: Transversus abdominis and obliquus internus activity during pilates exercises: measurement with ultrasound scanning. Arch Phys Med Rehabil, 2008, 89: 2205-2212. [Medline] [CrossRef]

10) Kiesel KB, Uhl TL, Underwood FB, et al.: Measurement of lumbar multifidus muscle contraction with rehabilitative ultrasound imaging. Man Ther, 2007, 12: 161-166. [Medline] [CrossRef]

11) de Paula Lima PO, de Oliveira RR, Costa LO, et al.: Measurement properties of the pressure biofeedback unit in the evaluation of transversus abdominis muscle activity: a systematic review. Physiotherapy, 2011, 97: 100-106. [Medline] [CrossRef]

12) Saliba SA, Croy T, Guthrie R, et al.: Differences in Transverse Abdominis Activation with Stable and Unstable Bridging Exercises in Individuals with Low Back Pain. N Am J Sports Phys Ther, 2010, 5: 63-73. [Medline]

13) Cholewicki J, McGill SM: Mechanical stability of the in vivo lumbar spine: implications for injury and chronic low back pain. Clin Biomech (Bristol, Avon), 1996, 11: 1-15. [Medline] [CrossRef]

14) Cholewicki J, Simons AP, Radebold A: Effects of external trunk loads on lumbar spine stability. J Biomech, 2000, 33: 1377-1385. [Medline] [CrossRef]

15) Hodges PW, Richardson CA: Inefficient muscular stabilization of the lumbar spine associated with low back pain. A motor control evaluation of transversus abdominis. Spine, 1996, 15: 2640-50. 